\title{
Chromatic Number, Independence Ratio, and Crossing Number
}

\author{
Michael O. Albertson \\ Department of Mathematics and Statistics \\ Smith College, Northampton, MA 01063, USA
}

Received 18 July 2007, accepted 29 November 2007, published online 17 June 2008

\begin{abstract}
Given a drawing of a graph $G$, two crossings are said to be dependent if they are incident with the same vertex. A set of crossings is independent if no two are dependent. We conjecture that if $G$ is a graph that has a drawing all of whose crossings are independent, then the chromatic number of $G$ is at most 5 . We show that this conjecture is true if the crossing number of $G$ is at most three. We also show that if all crossings are independent, then the chromatic number of $G$ is at most 6 , and the independence ratio of $G$ is at least $\frac{3}{16}$.
\end{abstract}

Keywords: Graphs, chromatic number, crossing number.

Math. Subj. Class.: 05C15, 05C10

\section{Introduction}

Progress towards the Four Color Theorem has had an enormous and continuing impact on the development of graph theory. One consequence is that it is compelling to consider whether a particular relaxation of planarity forces an upper bound on the chromatic number. We revisit the three classic generalizations of planarity. For more than a century there has been a coloring theorem for graphs embedded on surfaces of positive Euler genus. For almost forty years we have known that that coloring theorem is best possible. Restricting our attention to graphs of a given thickness, we know a relatively small interval in which the maximum chromatic number must live, though narrowing that interval appears difficult. Surprisingly, there are almost no results on coloring graphs with a given crossing number. The purpose of this paper is to provoke further work on the relationship between the crossing number and the chromatic number or especially the independence ratio. Towards this end we introduce a refinement to the idea of crossing and use this refinement to give bounds on these two

E-mail address: albertson@math.smith.edu (Michael O. Albertson) 
parameters for certain families of graphs. Section 2 provides the necessary background, mentions what has been done before, and develops some bounds. Section 3 gives results and proofs. We offer two explicit and many implicit open questions.

\section{Background}

Suppose $G$ is a graph with $n$ vertices and chromatic number $\chi(G)$. Recall that $U \subseteq V(G)$ is said to be independent if $x, v \in U \Rightarrow x v \notin E(G)$. The independence number of $G$, denoted by $\alpha(G)$, is the cardinality of a maximum independent set in $G$. The independence ratio, denoted by $\mu(G)$, is defined by $\mu(G)=\frac{\alpha(G)}{n}$. Since $\frac{n}{\chi(G)}$ is the average size of a color class in a $\chi(G)$-coloring of $G, \alpha(G) \geq \frac{n}{\chi(G)}$ and $\mu(G) \geq \frac{1}{\chi(G)}$. Results on the independence ratio of locally planar graphs (those embedded on a given surface with large (edge) width [2]) preceded and, in one sense, are stronger than the results about chromatic numbers for this same class of graphs. It is well known that locally planar graphs on a given surface are 5 -colorable $[4,6,12]$. The stronger result for independence ratios is given $\epsilon>0, \exists N(\epsilon, S)$ such that if $G$ is a graph with $n$ vertices embedded on $S$ and $n \geq N(\epsilon, S)$, then $\mu(G)>\frac{1}{4}-\epsilon[1,3]$. The earlier of the two papers just cited introduced the name independence ratio, although the idea had occurred prior to that e.g. in the work of Vizing and Plesnevič [13].

The crossing number of $G$, denoted by $c=\operatorname{cr}(G)$, is the minimum number of crossings in any nice drawing of $G$ in the plane. See [11] for a clear explanation of the foundational issues involved with crossings including the idea of a nice drawing. That paper also presents a survey of crossing numbers. A bibliography on crossing numbers is maintained in [14]. We will assume that our drawings are both nice and optimal with respect to the number of crossings.

Naturally, the first question to ask is whether the chromatic number is bounded from above by a function of the crossing number. To answer this question it is convenient to examine the incidence relation between vertices and crossings. Suppose $e=u v$ and $f=x y$ are two edges that cross in a given drawing of $G$. We say that $\{u, v, x, y\}$ is the cluster of vertices associated with the crossing $\{e f\}$, and the vertices of the cluster and the crossing are said to be incident with each other. The crossing covering number of a graph $G$, denoted by $\rho(G)$, is the minimum over all (both optimal and nice) drawings of $G$ of the minimum cardinality of a set of vertices subject to the condition that every crossing is incident with at least one vertex in the set.

Theorem 1. $\chi(G) \leq 4+\rho(G)$.

Proof. Given the drawing of $G$, let $C$ denote the set of vertices in a minimum crossing cover. Let $G_{0}$ denote the subgraph of $G$ induced by the vertices that are not in $C$. Since $G_{0}$ is planar, the vertices of $G_{0}$ can be colored using colors $1,2,3,4$. The vertices in $C$ can each be assigned a unique color using colors $5,6, \ldots, 4+\rho(G)$.

Noting that $\rho(G) \leq \operatorname{cr}(G)=c$ we immediately get.

Corollary 2. $\chi(G) \leq 4+c$.

Theorem 1 is best possible in the sense that for any integer $r \geq 0$, there exists a $(4+r)$ chromatic graph which has $\rho(G)=r$. To accomplish this join every pair of vertices in $C$. This will create an $r$-clique; however, $C$ is still a minimum crossing cover of the resulting 
graph. Then one needs to assure that $G_{0}$ is 4-chromatic and that every vertex in $C$ is adjacent to every vertex in $G_{0}$. Corollary 2 is best possible if $\operatorname{cr}(G) \leq 1$ but not so much for larger values of the crossing number. Since $\operatorname{cr}\left(K_{n}\right)=\Theta\left(n^{4}\right)$, one might expect the right bound in Corollary 2 to be $\mathrm{O}\left(c^{1 / 4}\right)$.

In the context of crossings and colorings the second natural question to ask would be what hypotheses on the crossings force the chromatic number to be relatively small. There have been a few minor results detailed below, and we will offer some more in the next section. However, the independence ratio may be a better measure of the near planarity of a given graph $G$ than its chromatic number. A drawing of a graph with a given number of crossings may have these crossings concentrated in a relatively small subgraph that has a relatively large chromatic number. Alternatively, it may have the crossings dispersed throughout the drawing and have relatively small chromatic number. In either case one expects that the independence ratio will be relatively large. We formalize this observation in the theorem below but emphasize that its interest is limited to instances where the number of vertices is much larger than the crossing number.

Theorem 3. Suppose $G$ has a drawing of a graph with $n$ vertices and c crossings. For any $\epsilon>0$, if $n \geq \frac{c}{4 \epsilon}$, then $\mu(G) \geq \frac{1}{4}-\epsilon$.

Proof. Let $G_{0}$ be the graph obtained by removing one vertex from each cluster of the drawing of $G$. Evidently, $G_{0}$ is planar, $\alpha(G) \geq \alpha\left(G_{0}\right) \geq \frac{n-c}{4}$, and the result follows.

We return to colorings. It is straightforward to show that if $\operatorname{cr}(G)=1$, then $\chi(G) \leq 5$. One way to see this is to apply Theorem 1 . Oporowski and Zhao proved the two results below and asked the open question that follows.

Theorem 4 ([7]). If $\operatorname{cr}(G) \leq 2$, then $\chi(G) \leq 5$.

Since $\operatorname{cr}\left(K_{6}\right)=3$ one cannot extend Theorem 4 without additional hypotheses. Let $\omega(G)$ denote the clique number, the cardinality of a largest clique contained in $G$.

Theorem 5 ([7]). If $\operatorname{cr}(G)=3$ and $\omega(G) \leq 5$, then $\chi(G) \leq 5$.

Let $K_{3} \vee C_{5}$ denote the join of triangle and a 5-cycle. Note that $\operatorname{cr}\left(K_{3} \vee C_{5}\right)=6, \chi\left(K_{3} \vee\right.$ $\left.C_{5}\right)=6$, and $\omega\left(K_{3} \vee C_{5}\right)=5$. This inspires the following.

Question 6 ([7]). If $\operatorname{cr}(G) \leq 5$ and $\omega(G) \leq 5$, is $\chi(G) \leq 5$ ?

We close this section by introducing a refinement to the notion of crossing that will force a coloring theorem.

Definition 7. In a drawing of a graph, two crossings are said to be dependent if their clusters have a common vertex. A set of crossings is said to be independent if no two are dependent.

\section{Graphs with Independent Crossings}

Theorem 8. If $G$ is a graph which has a drawing in the plane in which there are at most three crossings, no two of which are dependent, then $\chi(G) \leq 5$ and $\mu(G) \geq \frac{1}{5}$. 


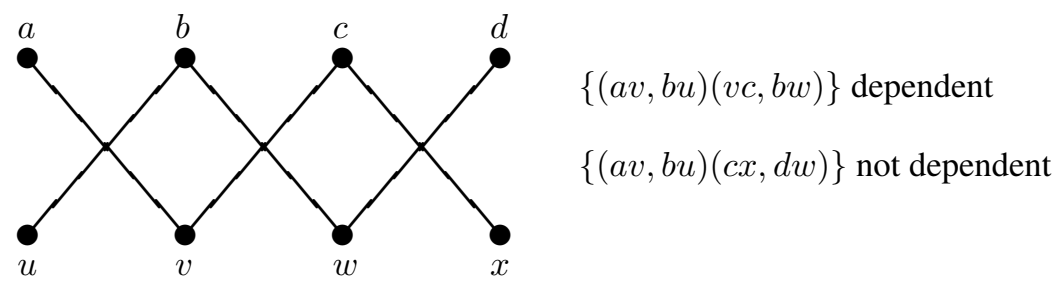

Figure 1: Dependent and Independent Clusters

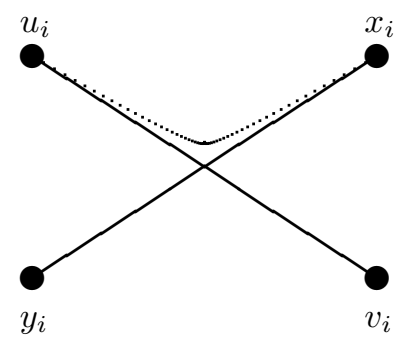

Figure 2: Clusters Induce $K_{4}$ 's

Proof. By Theorem 4 we may assume that there are exactly three crossings. For $1 \leq i \leq 3$, let $C_{i}=\left\{u_{i}, v_{i}, x_{i}, y_{i}\right\}$ denote the cluster of vertices associated with the $i^{\text {th }}$ crossing. By the independence of the crossings these vertices are all distinct. We assume that the vertices are labeled so that $u_{i} v_{i}$ crosses $x_{i} y_{i}$. We may assume that each $C_{i}$ induces a $K_{4}$. If it does not, we can add edges to the cluster without creating any additional crossings by joining e.g. $u_{i}$ to $x_{i}$, with an edge that is drawn within a small neighborhood of the crossing pair of edges. This is illustrated with the dotted edge in the figure below.

Consider $G_{0}=G\left[\cup_{j=1}^{3} C_{j}\right]$, the subgraph of $G$ induced by the clusters $C_{1}, C_{2}$, and $C_{3}$. $\left|V\left(G_{0}\right)\right|=12$. Since $G_{0}$ has three independent crossings, Euler's formula implies that $\left|E\left(G_{0}\right)\right| \leq 33$. Since we are assuming each cluster contains 6 edges, there are at most 15 edges joining vertices in one cluster with vertices in another.

Claim 9. $\exists Z=\left\{z_{1}, z_{2}, z_{3}: z_{i} \in C_{i}\right.$ and $Z$ is an independent set $\}$.

Proof of claim. Since there are four choices for each $z_{i}$, there exist 64 candidates for $Z$. If the claim fails for a particular candidate set, there must be at least one edge joining two of the three vertices of $Z$. Each edge joining two vertices in different clusters is contained in 4 candidate sets. Thus, if the claim fails there must be at least 16 edges joining vertices in different clusters. Since there are at most 15 such edges, the claim holds.

Continuing the proof of Theorem 8, given the independent set $Z=\left\{z_{1}, z_{2}, z_{3}\right\}$ for $1 \leq$ $i \leq 3$ let $e_{i}$ denote the crossing edge in $C_{i}$ that is incident with $z_{i}$. Set $G_{1}=G-\left\{\cup_{i=1}^{3} e_{i}\right\}$. Since $G_{1}$ is planar, we 4 -color $G_{1}$ using colors $\{1,2,3,4\}$. We then transfer this coloring to $G$ and recolor each $z_{i}$ with the color 5 . 
A probabilistic argument for the claim in the above theorem was also given by Michael Sindelair [9].

Although the proof above does not generalize to more than three crossings, the following conjecture is appealing.

Conjecture 10. If $G$ is a graph which has a drawing in the plane in which no two crossings are dependent, then $\chi(G) \leq 5$ and $\mu(G) \geq \frac{1}{5}$.

Both Stephens [10] and the author noted that there is a straightforward argument that shows that graphs all of whose crossings are independent are 8-colorable. The following slightly stronger result generalizes.

Theorem 11. If $G$ is a graph which has a drawing in the plane in which no two crossings are dependent, then $\chi(G) \leq 7$ and $\mu(G) \geq \frac{1}{7}$.

Proof. If $G_{1}$ is an induced subgraph of $G$ with $n_{1}$ vertices, then in the restriction of the drawing of $G$ to the subgraph $G_{1}$, the number of crossings is at most $\frac{n_{1}}{4}$. Thus there are at most $\frac{n_{1}}{4}$ edges whose removal will make a subgraph of $G_{1}$ planar. Consequently, $\left|E\left(G_{1}\right)\right| \leq$ $3 n_{1}-6+\frac{n_{1}}{4}$, and the average degree in $G_{1}$ is less than 7 . The standard greedy coloring of $G$ will use no more than seven colors.

A straightforward extension of the preceding argument yields the following result.

Theorem 12. If $G$ is a graph which has a drawing in the plane in which every vertex is incident with at most $r$ crossings, then $\chi(G) \leq 6+\left\lceil\frac{r}{2}\right\rceil$.

Now we get one step closer to the conjecture.

Theorem 13. If $G$ is a graph which has a drawing in the plane in which no two crossings are dependent, then $\chi(G) \leq 6$ and $\mu(G) \geq \frac{1}{6}$.

Proof. Suppose there are $r$ crossings whose vertices form clusters $C_{1}, \ldots C_{r}$ with $C_{i}=$ $\left\{x_{i}, y_{i}, u_{i}, v_{i}\right\}$ such that $x_{i} y_{i}$ and $u_{i} v_{i}$ cross. Let $G_{1}=G-\left\{\cup_{j=1}^{r} x_{j} y_{j}\right\}$. Since we have removed a crossing edge from each induced cluster, $G_{1}$ is planar. Let $\gamma_{1}$ be a 4-coloring of $G_{1}$ using colors from $\{1,2,3,4\}$. As in the proof of Theorem 8 we may assume that each $C_{i}$ induces a $K_{4}$ in $G$. This implies that each $C_{i}$ induces a $K_{4}-e$ in $G_{1}$. Thus $\gamma_{1}$ necessarily assigns at least three different colors to the vertices of $C_{i}$. For $1 \leq i \leq r$ we choose $z_{i} \in C_{i}$ such that $\gamma_{1}\left(z_{i}\right) \in\{1,2\}$ and $w_{i} \in C_{i}$ such that $z_{i} w_{i}$ is a crossing edge in $C_{i}$.

Now define $G_{2}=G-\left\{\cup_{j=1}^{r} z_{j} w_{j}\right\}$. Since we have removed a crossing edge from each cluster $G_{2}$ is planar. Let $\gamma_{2}$ be a 4-coloring of $G_{2}$ using colors from $\{1,2,3,4\}$. Let $\gamma$ be a coloring of $G$ defined as follows. For $x \neq z_{i}, 1 \leq i \leq r$, set $\gamma(x)=\gamma_{2}(x)$. For $1 \leq i \leq r$, set $\gamma\left(z_{i}\right)=\gamma_{1}\left(z_{i}\right)+4$. It is straightforward to check that $\gamma$ is a 6 -coloring of $G$.

Note that if an edge in a drawing of $G$ crosses two other edges, then these two crossings are dependent. Thus the hypothesis of no two dependent crossings implies that $G$ can be drawn in the plane so that no edge crosses more than one other edge i.e. $G$ is 1-embeddable in the plane. Consequently, Theorem 13 could be achieved as a corollary of Borodin's Theorem [5]; however, Borodin's result, applying to a larger class of graphs, seems to require a difficult proof.

Theorem 14. If $G$ is a plane graph which has a drawing in the plane in which no two crossings are dependent, then $\mu(G) \geq \frac{3}{16}$. 
Proof. If all crossings are independent, then $\operatorname{cr}(G) \leq \frac{n}{4}$. By choosing one vertex from each cluster we obtain $U \subset V(G)$ such that $|U| \leq \frac{n}{4}$ and $G_{1}=G[V-U]$ is planar. Using the Four Color Theorem gives that $\alpha(G) \geq \alpha\left(G_{1}\right) \geq \frac{1}{4} \cdot \frac{3 n}{4}=\frac{3 n}{16}$.

Repeating a theme mentioned in the preceding section, the proof of this independence ratio result is easier than the proof of the coloring result, even though the independence result is, in one sense, stronger.

Postscript: With respect to the discussion following Corollary 2, Marcus Schaefer has shown that if $c=\operatorname{cr}(G)$, then $\chi(G)=\mathrm{O}\left(c^{1 / 4}\right)[8]$.

\section{References}

[1] Michael O. Albertson and Joan P. Hutchinson, On the independence ratio of a graph, J. Graph Theory 2 (1978), no. 1, 1-8.

[2] Michael O. Albertson and Joan P. Hutchinson, The independence ratio and genus of a graph, Trans. Amer. Math. Soc. 226 (1977), 161-173.

[3] Michael O. Albertson and Joan P. Hutchinson, The maximum size of an independent set in a nonplanar graph, Bull. Amer. Math. Soc. 81 (1975), 554-555.

[4] Michael O. Albertson and Walter R. Stromquist, Locally planar toroidal graphs are 5-colorable, Proc. Amer. Math. Soc. 84 (1982), no. 3, 449-457.

[5] Oleg Borodin, A new proof of a 6-color theorem, J. Graph Theory 19 (1995), 507-521.

[6] Joan P. Hutchinson, A five-color theorem for graphs on surfaces, Proc. Amer. Math. Soc. 90 (1984), no. 3, 497-504.

[7] Bogdan Oporowski and David Zhao, Coloring graphs with crossings, preprint.

[8] Marcus Schaefer, personal communication.

[9] Michael Sindelair, personal communication.

[10] D. Christopher Stephens, personal communication.

[11] László Székely, A successful concept for measuring non-planarity of graphs: the crossing number, Discrete Math. 276 (2004), no. 1-3, 331-352.

[12] Carsten Thomassen, Five-coloring maps on surfaces, J. Combinatorial Theory Series B 59 (1993), 89-105.

[13] V. G. Vizing and G. S. Plesnevič, On the problem of the minimal coloring of the vertices of a graph, in Russian, Sibirsk. Mat. Ž 6 (1965) 234-236.

[14] Imrich Vrt'o, Crossing Numbers of Graphs: A Bibliography, ftp://ftp.ifi.savba.sk/pub/imrich/crobib.pdf 